\title{
Emotion-Driven Interactive Digital Storytelling
}

\author{
Huiwen Zhao ${ }^{1}$, Jian J Zhang ${ }^{1}$, and Siné McDougall ${ }^{2}$ \\ ${ }^{1}$ National Center for Computer Animation \\ Bournemouth University \\ Fern Barrow, Poole, Dorset, BH12 5BB, United Kingdom \\ \{hwzhao, jzhang\} @bournemouth.ac.uk \\ ${ }^{2}$ Design, Engeering and Computing \\ Bournemouth University \\ Fern Barrow, Poole, Dorset, BH12 5BB, United Kingdom \\ \{smcdougall\} @bournemouth.ac.uk
}

\begin{abstract}
Interactive digital storytelling has attracted a great deal of research interest in recent years. However, most interactive stories are told following a goal-oriented and task-based mode, which motivates the player to interact with stories by achieving the goals rather than empathizing with the characters and experience the enriched emotions. Given this fact, we propose an emotion-driven interactive digital storytelling approach based on Smith and Lazarus' cognitive theory of emotion. In this approach, the player's emotions, as a driving force, motivate the story forward and contribute to their experience directly and explicitly. To evaluate this approach, an interactive video was made by re-editing existing footage of the TV comedy Ugly Betty and ten players were interviewed afterwards. The results reveal that the interviewees' experience is largely influenced by their gender and favorite media entertainment.
\end{abstract}

Keywords: interactive digital storytelling, emotion-driven, cognitive theory of emotion, user experience.

\section{Introduction}

Interactive digital storytelling, as a promising entertainment industry, has attracted a great deal of research interest in recent years. Most interactive digital storytelling systems to date employ a goal-oriented and task-based approach. That is to say, the way that a story evolves is driven by the goals. In order to achieve a goal, a set of tasks are structured by generating a sequence of actions [1]. Therefore, the motivation of interacting with stories primarily comes from competing with others to achieve these goals rather than empathizing with the characters' experience to enrich emotions and guide story development accordingly. Interactive digital storytelling therefore ignores the capacity of empathized emotions in creating an engaging story to contribute to users' experience. It does not satisfy potential players who are oriented to movies [2] and demographic groups who are interested in attractive and challenging stories [3] [4]. Given this consideration, we propose a new design approach-emotion-driven interactive digital storytelling - in which players experience a series of emotions by empathizing with the characters, more importantly, the players' emotions act as the 
explicit driving force to motivate the story forward. The aim of the emotion-driven interactive digital storytelling approach is to allow players to adapt narrative storylines in accordance with their experienced emotions.

\section{Emotion-Driven Interactive Digital Storytelling}

\subsection{Cognitive-Motivational-Emotive Theory}

Emotions play a significant role in a wide range of human cognitive processes. A number of cognitive theories of emotion have emerged accordingly. Appraisal theories which argue that emotions arise from two basic processes: appraisal and coping are widely applied into several computational models of emotion, for example, EMA [5], FLAME [6], WILL [7]. In particular, our work is inspired by Smith and Lazarus' [8] cognitive-motivational-emotive theory.

In a classic paper, Smith and Lazarus examined how emotions arise in particular situations through an appraisal process and how individuals are likely to respond given their current needs and goals as well as their longer term attitudes and beliefs. Depending on their personality, two individuals can react with quite similar or entirely different emotions towards the same situation through different appraisal processes. The emotions individuals experience lead to an action tendency which is an urge to respond to the situation in a particular way. The nature of the emotions determines the nature of action tendency which leads to different coping strategies, for example, to attack in anger, flee or avoid in anxiety. Influenced by these coping strategies, action tendencies are translated to a wide array of behavioral responses or actions. Smith and Lazarus argued that our experience of emotions and our responses to them changed dynamically with the situation we found ourselves in. Furthermore, they argued behavioral responses would not stop the emotional experience, but continuously influence the subsequent appraisals and emotions leading to new actions.

\subsection{Emotion-Driven Interactive Digital Storytelling}

When Smith and Lazarus' theory is applied to interactive digital storytelling, emotions can act as the driving force to motivate narrative evolution. As an important mechanism of emotional involvement with narrative, empathy helps the player build emotional bond with the character [9]. By thinking and feeling from the character's perspective, the player experiences the character's emotions vicariously. Due to the influence of personality on the appraisal process, each player might feel different emotions. Therefore, in emotion-driven interactive digital storytelling, the players are given the opportunity to make a series of choices as they move through the narrative about how they feel from the character's perspective in a variety of emotional conflict moments. The choices they make determine the character's emotions and then result in different action tendencies as well as behavioral responses in the character. In addition, the character's actions with respect to a narrative can also serve as stimuli to evoke new emotions in the player and then trigger new actions. Therefore, the choices players make which reflect their emotional reactions towards the storyline can result in a particular series of events being shown which, in turn, evoke new emotions and trigger new actions in interactive digital storytelling. The series of emotional responses of the 
player direct the narrative and determine the final 'story' they experience. In this sense, different players would experience different stories based on their different emotions evoked by empathizing with the characters. Emotions, thus, become a powerful driving force for narrative configuration in interactive digital storytelling [10].

\section{Evaluating Emotion-Driven Interactive Digital Storytelling}

\subsection{Method}

Semi-structured interviews were used to elicit information about the player's experience, especially with respect to their emotional engagement and enjoyment. Questions were designed to prompt the interviewee's experience regarding their narrative understanding, emotional involvement, emotion-driven interaction and overall enjoyment based on the theory of narrative engagement [11].

\subsection{Materials}

To focus on the narrative aspect and reduce the influence of gameplay as well as other technical issues, an interactive video was made to evaluate user experience by re-editing existing footage from the TV comedy Ugly Betty creating a series of storyline segments which could be pieced together in different ways rather than shooting each segment from scratch. The method used to capture players' emotions was similar to that employed by Roberts et al. [12], namely, players were asked questions about their emotions after each video segment. Their responses at these points determined the main character Betty's actions. Therefore, the video segment which contained these actions would be chosen from a series of options and shown to the player next.

\subsection{Participants and Procedure}

Ten students and staff ( 5 male and 5 female) participated in this study. All of them were English speakers. Every interviewee was required to watch the interactive video individually. The video was shown online. Before they started to watch the video, an introductory text on the homepage was shown to explain the procedure of this study and how to interact with the video. After watching the video, a demographic questionnaire was required to fill out which aimed to get their background information, such as their favorite entertainment, whether they have watched Ugly Betty before. Following the demographic questionnaire, the interviews were conducted. All interviews were recorded and transcribed.

\subsection{Results}

We found interviewees' experiences were largely influenced by gender and their favorite media entertainment. In general, females felt more engaged than males with the emotional involvement and emotion-driven interaction. All females felt empathy for, and built the emotional bond with, the character. They perceived the emotions from the character's standpoint while at the same time combining this with their own experience and concerns. They made the choices based on the blend of these two emotions. 
"Sometimes it was my own emotional sort of connection to the story, sometimes sort of putting (myself) in her issues...."

In contrast, male interviewees found it relatively difficult to empathize with Betty. Although they enjoyed this interactive experience, their motivations in making choices were made more on the basis of their own feelings or self-set goals rather than emotional empathy with the character.

"I have a goal of bringing this guy down ... I invented this goal and gave it to myself. My personal emotion was affected both by what I was looking at and by whether I've achieved my goal or not."

Two other factors determined the interviewees' user experience: their previous interactive digital storytelling experience and their favorite media entertainment. Interviewees, who had interactive digital storytelling experience before, especially those who prefer digital games, felt the interaction in this video was "slow". They expected more choices and shorter video segments. Influenced by the mode of interaction with digital games, one interviewee said:

"I was expecting like in games that I would have choices very earlier on and much more often."

For other interviewees who liked watching TV or film, the number of choices was not a problem. They enjoyed watching the interactive video in the same way as watching a TV program or film while also enjoying the feeling of influencing how the story evolved.

"I think it (the length of the video segments) is alright, because you tell a story. For me, it is like a movie story."

Because existing TV footage was used, the player's experience is also affected by the original storyline of Ugly Betty. The interactive video creates multiple choices and storylines. To do this the temporal and spatial structure of original linear TV footage is broken and recomposed. Visual and sound discontinuities which make the story difficult to understand may therefore result. Fortunately, this was not reflected in interviewees' reports. Only one interviewee pointed out that one transition between two video segments was not smooth, but it did not affect his understanding of the whole story. Almost all interviewees expressed their interest in watching other storylines even those who had watched the original Ugly Betty before.

\section{Discussion and Future Work}

The interviews reveal that the user experience of this emotion-driven interactive video is impacted by the different player types (i.e. male vs female) and their preferences for different media (digital games vs traditional narrative media). In general, females feel more emotional involvement and empathy than males and those who like traditional 
media entertainment, such as TV and film, get more pleasure from interaction than those who like playing digital games: importantly, these findings have since been confirmed in convergent quantitative research carried out in our laboratory. These two factors are inter-related. Usually traditional media entertainment tends to create other-directed emotions, i.e. emotions directed towards others through empathy. In contrast, digital games are more likely to evoke self-directed emotions which concern the player's success and desires to fulfill their goals or tasks [13]. Psychological research [14][15][16] has shown that women are more likely to feel and express other-directed emotions than men who are more likely to experience self-directed emotions. This may be the reason why women tend to be the predominant audience of TV dramas since they focus on the emotional relations between the main protagonists [17], while males are dominant in the world of digital games [18]. Females therefore appear to be the potential target audience for this emotion-driven interactive digital storytelling.

This study also indicates that re-editing television footage can provide an effective way of making interactive stories, it is clear that the choice of footage is critical. The TV comedy Ugly Betty appears to cater more to women's taste, so it may be possible to choose material which might engage men better. For example, one player said he would be more interested if "there was Arnold Schwarzenegger". In addition to making characters more relevant, other footage could alter the pace and narrative style of the original material to make it faster moving. However, it is important to note that it takes time to create emotional conflicts and evoke emotions. This leads to a conflict between the length of video and the frequency of the player's interactions: further research is required to determine how long a video segment should be to evoke sufficient emotions while maintaining the player's interest in interaction.

In future, we will examine in more detail how different types of players and their previous experience determines their emotional response to storytelling to provide information which will make it possible to predict which target groups are likely to enjoy emotion-driven interactive digital storytelling. Two main concerns became apparent from this work: the need to find appropriate narrative genres for different groups of individuals and solving the conflict between the length of the video segment and the frequency of the player's interactions. Nevertheless, the results suggest that this emotion-driven interactive digital storytelling can create a variety of different emotional user experiences and has the potential to develop as a new approach for interactive digital storytelling design.

Acknowledgements. I would like to show my gratitude to Dr. Jim Pope. This paper would not have been possible without his guidance and help.

\section{References}

1. Pizzi, D., Cavazza, M.A.: Affective storytelling based on characters' feelings. In: Intelligent Narrative Technologies: Papers from the AAAI Fall Symposium, pp. 111-118 (2007)

2. Louchart, S., Aylett, R., Kriegel, M., Dias, J., Figueiredo, R., Paiva, A.: Authoring emergent narrative-based games. Journal of Game Development 3(1), 19-37 (2008)

3. Duh, H.B., Yee, S.L.C.Y., Gu, Y.X., Chen, V.H.: A narrative-driven design approach for casual games with children. In: Sandbox 2010, Los Angeles, California, July 28-29 (2010) 
4. Hartmann, T., Klimmt, C.: Gender and Computer Games: Exploring Females' Dislikes. Journal of Computer-Mediated Communication 11 (2006)

5. Marsella, S., Gratch. J.: Modeling coping behavior in virtual humans: don't worry, be happy. In: AAMAS, pp. 313-320 (2003)

6. El-Nasr, M.S., Yen, J., Ioerger, T.: FLAME: Fuzzy Logic Adaptive Model of Emotions. In: Autonomous Agents and Multi-Agent Systems, vol. 3, pp. 219-257 (2000)

7. Moffat, D., Frijda, N.: Where there's a Will there's an agent. In: Presented at Workshop on Agent Theories, Architectures and Languages (1995)

8. Smith, C.A., Lazarus, R.S.: Emotion and Adaptation. In: Pervin, L.A. (ed.) Handbook of Personality: Theory and Research, pp. 609-637 (1990)

9. Zillmann, D.: Mechanisms of emotional involvement with drama. Poetics 23(1-2), 33-51 (1994)

10. Nath, S.: Narrativity in User Action: Emotion and Temporal Configurations of Narrative. In: Proceedings of the 4th International Conference on Computational Semiotics for Games and New Media (COSIGN 2004), Split, Croatia (2004)

11. Busselle, R., Bilandzic, H.: Measuring Narrative Engagement. Manuscript in Press in Media Psychology (2009)

12. Roberts, D., Narayanan, H., Isbell, C.: Learning to Influence Emotional Responses for Interactive Storytelling. In: Proceedings of the 2009 AAAI Symposium on Intelligent Narrative Technologies II (2009)

13. Ryan, M.L.: Interactive Narrative, Plot Types, and Interpersonal Relations. In: Proceedings of the 1st Joint International Conference on Interactive Digital Storytelling (2008)

14. Johnson, J., Shulman, G.: More alike than meets the eye: Perceived gender differences in subjective experience and its display. Sex Roles 19, 67-79 (1988)

15. Stoppard, J., Grunchy, C.G.: Gender, context and expression of positive emotions. Personality and Social Psychology 64, 211-220 (1993)

16. Brody, L.: Beyond Stereotypes: Gender and emotion. Journal of Social Issues 53, 369-393 (1997)

17. McQuail, D.: Mass Communication Theory: An Introduction. Sage, London (1994)

18. Lucas, K., Sherry, J.: Sex Differences in Video Game Play: A communication-Based Explanation. Communication Research 31(5), 499-523 (2004) 